Research Article

\title{
Hypertension Subtypes among Thai Hypertensives: An Analysis of Telehealth-Assisted Instrument in Home Blood Pressure Monitoring Nationwide Pilot Project
}

\author{
Sakolwat Montrivade, ${ }^{1}$ Pairoj Chattranukulchai $\left(\mathbb{D},{ }^{1}\right.$ Sarawut Siwamogsatham $\left(\mathbb{D},{ }^{2}\right.$ \\ Yongkasem Vorasettakarnkij, ${ }^{2}$ Witthawat Naeowong, ${ }^{2}$ Patchaya Boonchayaanant $\mathbb{D},{ }^{3}$ \\ Anut Sakulsupsiri, ${ }^{4}$ Aekarach Ariyachaipanich, ${ }^{1}$ Vorarit Lertsuwunseri, ${ }^{1}$ \\ Voravut Rungpradubvong, ${ }^{1}$ Sudarat Satitthummanid, ${ }^{1}$ Sarinya Puwanant, ${ }^{1}$ \\ Somchai Prechawat, ${ }^{1}$ Suphot Srimahachota, ${ }^{1}$ Jarkarpun Chaipromprasit, ${ }^{1}$ \\ Wacin Buddhari, ${ }^{1}$ Smonporn Boonyaratavej, ${ }^{1}$ Surapun Sitthisook, ${ }^{1}$ \\ Peera Buranakitjaroen, ${ }^{5}$ Apichard Sukonthasarn, ${ }^{6}$ and Somkiat Sangwatanaroj ${ }^{1}$ \\ ${ }^{1}$ Division of Cardiovascular Medicine, Chulalongkorn University, King Chulalongkorn Memorial Hospital, \\ 10330 Bangkok, Thailand \\ ${ }^{2}$ Division of Hospital and Ambulatory Medicine, Chulalongkorn University, King Chulalongkorn Memorial Hospital, \\ 10330 Bangkok, Thailand \\ ${ }^{3}$ Division of Endocrinology and Metabolism, Department of Medicine, Faculty of Medicine, Chulalongkorn University, \\ King Chulalongkorn Memorial Hospital, 10330 Bangkok, Thailand \\ ${ }^{4}$ Pharmacy Department, King Chulalongkorn Memorial Hospital, 10330 Bangkok, Thailand \\ ${ }^{5}$ Department of Medicine, Siriraj Hospital, Mahidol University, 10700 Bangkok, Thailand \\ ${ }^{6}$ Thai Hypertension Society, 10310 Bangkok, Thailand
}

Correspondence should be addressed to Pairoj Chattranukulchai; pairoj.md@gmail.com

Received 2 October 2019; Revised 6 February 2020; Accepted 19 February 2020; Published 9 April 2020

Academic Editor: Tomohiro Katsuya

Copyright (C) 2020 Sakolwat Montrivade et al. This is an open access article distributed under the Creative Commons Attribution License, which permits unrestricted use, distribution, and reproduction in any medium, provided the original work is properly cited.

Background. White-coat hypertension (HT), masked HT, HT with white-coat effect, and masked uncontrolled HT are wellrecognized problems of over- and undertreatment of high blood pressure in real-life practice. However, little is known about the true prevalence in Thailand. Objectives. To examine the prevalence and characteristics of each HT subtype defined by mean home blood pressure (HBP) and clinic blood pressure (CBP) using telemonitoring technology in Thai hypertensives. Methods. A multicenter, observational study included adult hypertensives who had been diagnosed for at least 3 months based on CBP without the adoption of HBP monitoring. All patients were instructed to manually measure their HBP twice a day for the duration of at least one week using the same validated automated, oscillometric telemonitoring devices (Uright model TD-3128, TaiDoc Corporation, Taiwan). The HBP, CBP, and baseline demographic data were recorded on the web-based system. HT subtypes were classified according to the treatment status, CBP ( $\geq$ or $<140 / 90 \mathrm{mmHg}$ ), and mean HBP ( $\geq$ or $<135 / 85 \mathrm{mmHg}$ ) into the following eight subtypes: in nonmedicated hypertensives, there are four subtypes that are normotension, white-coat HT, masked HT, and sustained HT; in treated hypertensives, there are four subtypes that are well-controlled HT, HT with white-coat effect, masked uncontrolled HT, and sustained HT. Results. Of the 1,184 patients (mean age $58 \pm 12.7$ years, $59 \%$ women) from 46 hospitals, 1,040 $(87.8 \%)$ were taking antihypertensive agents. The majority of them were enrolled from primary care hospitals $(81 \%)$. In the nonmedicated group, the prevalence of white-coat and masked HT was $25.7 \%$ and $7.0 \%$, respectively. Among the treated patients, the HT with white-coat effect was found in $23.3 \%$ while $46.7 \%$ had uncontrolled HBP (a combination of the masked uncontrolled HT (9.6\%) and sustained HT (37.1\%)). In the medicated older subgroup $(n=487)$, uncontrolled HBP was more prevalent in male than in female $(53.6 \%$ vs. $42.4 \%, p=0.013)$. Conclusions. This is the first nationwide study in Thailand to examine the prevalence of HT subtypes. Almost one-fourth had white-coat HT or HT with white-coat effect. Approximately half of the treated patients 
especially in the older males had uncontrolled HBP requiring more intensive interventions. These results emphasize the role of HBP monitoring for appropriate HT diagnosis and management. The cost-effectiveness of utilizing THAI HBPM in routine practice needs to be examined in the future study.

\section{Introduction}

Hypertensive patients can be divided into several subtypes based on clinic blood pressure (CBP) and out-of-office blood pressure values including white-coat hypertension (HT), masked HT and sustained HT in nonmedicated patients or HT with white-coat effect, masked uncontrolled HT, and sustained HT in patients receiving antihypertensive medications [1-3]. However, the diagnosis is very challenging and is often overlooked since it requires both CBP and out-ofoffice BP data [4]. Previous studies reported the prevalence of white-coat HT and masked HT as high as $35 \%$ and $10 \%$, respectively $[2,3,5-7]$. In patients with white-coat HT and HT with white-coat effect, the overintensification of antihypertensive medications could potentially cause hypotension and worsen cardiovascular outcomes especially in the elderly $[8,9]$. On the other hand, patients with masked HT and masked uncontrolled HT may be at an increased risk of stroke comparable to those in patients with sustained HT [10]. Out-of-office BP measurement is crucial to confirm the diagnosis and to titrate BP-lowering medications in the patient with these HT subtypes [11, 12]. Home blood pressure monitoring (HBPM) is recommended by recent several guidelines [13-15] as a practical modality to obtain out-of-office BP, which is less expensive, less complex, and more widely available than ambulatory blood pressure monitoring (ABPM) [16-19]. The adoption of telemonitoring strategy, an Internet-based transmission system, allows linking home blood pressure (HBP) records between multiple HBPM devices and a central computer at the clinic. The data can be monitored and analyzed by trained healthcare professionals remotely and can facilitate improvement in managing hypertensive patients [11, 20-22]. This technology overcomes the self-reporting bias which is a limitation of HBPM in a clinical practice [23] since the HBP data transferring is completed without manual data entry by the patient.

The telehealth-assisted instrument in home blood pressure monitoring (THAI HBPM) project was designed to be a proof-of-concept observational multicenter study implementing the web-based telemonitoring. We aimed to examine the prevalence and characteristics of HT subtypes defined by mean HBP and CBP in real-life clinical setting across Thailand.

\section{Materials and Methods}

2.1. Study Oversight. THAI HBPM is a nationwide prospective observational study involving 46 centers across all regions of Thailand (see Supplementary Material for the details of all participating sites). The Ministry of Public Health of Thailand promoted the nationwide project and approved the study protocol and the centralized institutional review board review process.

2.2. Patient Population. Eligible participants were consecutively enrolled from 46 centers throughout the country. Adult patients who were 18 years of age or older with known HT diagnosed for more than 3 months based on CBP without adoption of HBPM were enrolled. If the participants were taking antihypertensive agents, they must have been on a stable dose of medications for at least 3 months before the enrollment. Patients with incomplete clinical characteristics or BP data will be excluded.

\subsection{Clinic and Home Blood Pressure Measurement. Clinic BP} was measured by trained healthcare professionals using the validated sphygmomanometer, after the patient had been resting in a relaxed, seated position $[24,25]$. We used the average of two consecutive readings at a 2-minute interval taken from the arm with the higher BP for the analysis.

Clinical validation between $\mathrm{CBP}$ and $\mathrm{HBP}$ readings was performed at the clinic according to the standard recommendation [26] before starting the HBP recording (day 0). The device is validated if there are less than $5 \mathrm{mmHg}$ differences of both SBP and DBP between sphygmomanometers and HBPM devices [26, 27] (see Supplementary Table 1).

Home BP data were obtained using the same validated automated, oscillometric devices (Uright model TD-3128, TaiDoc Technology Corporation, Taiwan, see Supplementary Figure 1). Trained healthcare providers instructed the participants to self-record HBP twice a day (1 hour after waking in the morning before taking antihypertensive medications or having breakfast and 30 minutes before going to bed) after 3 minutes of rest in a sitting position with two consecutive measurements, 1 minute apart for each recording. Blood pressure measurement continued for at least 7 days as recommended by standard guidelines [2, 28] during a 30-day period. In case there is a significantly different BP between both arms as determined at the enrollment visit, participants were instructed to use the arm with the greater BP. To avoid self-reporting bias, HBP values were automatically recorded in device memory. All participants were informed to bring the HBPM device along with them on the appointed clinic visit. At the $1^{\text {st }}$ follow-up visit (day 30-45), all recorded HBP data were transferred from the devices via USB cable to the Windows-based computer at the participating clinics. The data will then be automatically forwarded to cloud storage through the Internet-based transmission system. When HBP data had been uploaded, they could be viewed and analyzed using a regular Internet Explorer program via Uright Telehealth website (Figure 1). 


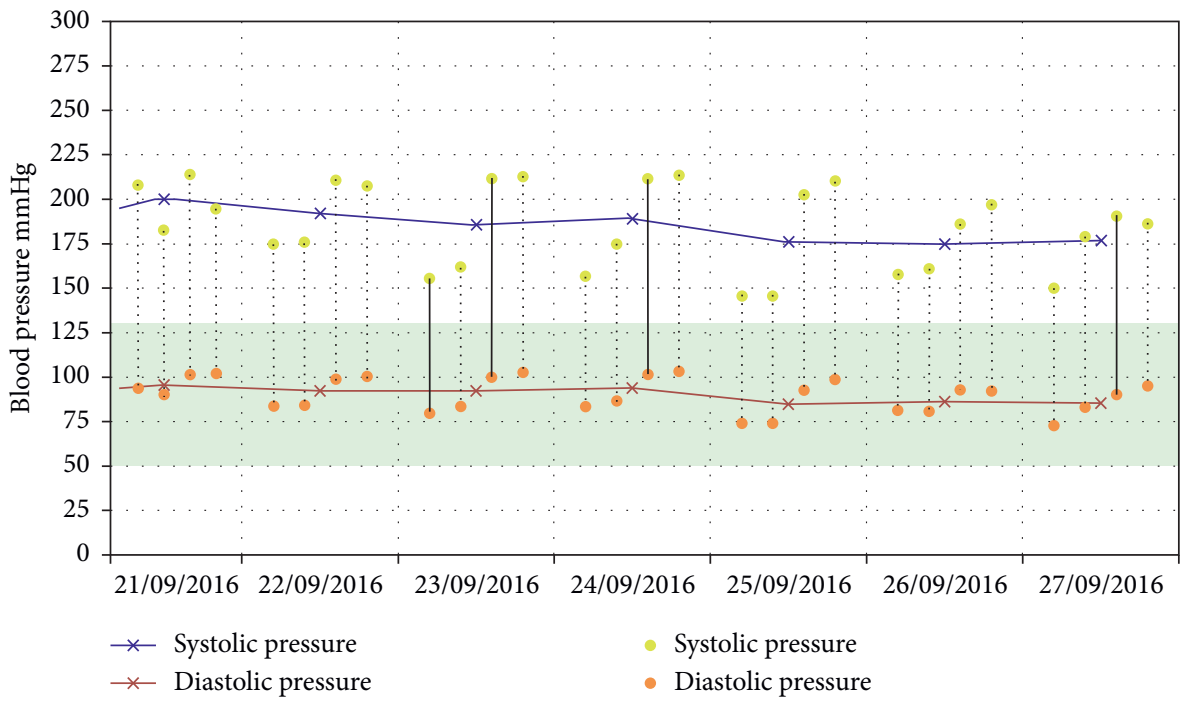

FIGURE 1: Example of consecutive 7-day home blood pressure (HBP) data viewed on the desktop computer via Uright Telehealth website of a 55 -year-old woman with a 2-year history of HT. The mean HBP was 184/94 mmHg while her clinic BP was 185/90 mmHg; hence, this BP pattern was categorized as sustained HT. Purple crosses represent daily average systolic HBP. Red crosses represent daily average diastolic HBP.

The device and Uright telehealth system were validated and approved by the US FDA [29]. A trained investigator at King Chulalongkorn Memorial Hospital who was blinded to the study demographic data independently interpreted the BP pattern. Patients who had at least 7-day HBP records will be included in the analysis. We discarded the measurements taken on the first day and used the mean value of all the remaining HBP records for the data analysis [16]. The CBP, demographic data, medical history, biochemistry laboratory results, and current antihypertensive medications were recorded on the web-based system.

2.4. Data Analysis. Participants were categorized according to the treatment status, CBP ( $\geq$ or $<140 / 90 \mathrm{mmHg}$ ), and HBP data ( $\geq$ or $<135 / 85 \mathrm{mmHg}$ ) into the following 8 subtypes $[2,28,30]$. In nonmedicated patients, there were 4 subtypes: (1) normotension: nonhypertensive CBP and HBP levels; (2) white-coat HT: hypertensive CBP level and nonhypertensive HBP level; (3) masked HT: nonhypertensive CBP level and hypertensive HBP level; and (4) sustained HT: both hypertensive CBP and HBP levels. Treated participants were categorized into another 4 subtypes: (5) well-controlled HT: nonhypertensive CBP and HBP levels; (6) HT with whitecoat effect: hypertensive CBP level and nonhypertensive HBP level; (7) masked uncontrolled HT: nonhypertensive CBP level and hypertensive HBP level; and (8) sustained HT: both hypertensive CBP and HBP levels.

Regarding the HBP control status in treated patients, the "controlled HBP group" consists of patients with wellcontrolled HT and HT with white-coat effect while the "uncontrolled HBP group" includes 2 other subtypes: masked uncontrolled HT and sustained HT. The white-coat effect (CBP and HBP difference) was calculated by mean CBP minus mean HBP (mmHg). Subgroup analyses were analyzed according to the country's regions, gender, and age ( $<60$ years and $\geq 60$ years).

Categorical variables were described as numbers $(n)$ and percentage of frequencies (\%). Continuous variables were shown as mean values and SD. Chi-square test and ANOVA were used for the analysis of categorical and continuous variables, respectively. We used SPSS software, version 22.0 (IBM) for statistical analysis.

\section{Results}

3.1. Patient Characteristics. A total of 1,250 patients were consecutively enrolled between August 2016 and August 2017. Of these, 66 were excluded due to incomplete clinical characteristics or BP data. Thus, 1,184 patients from 46 hospitals (5 regions: North, Northeast, East, Center, and South) were included in the analysis (see patient enrollment flow chart in Supplementary Figure 2). Patient characteristics are summarized in Table 1 . The mean $( \pm S D)$ age of the patients was $58.2 \pm 12.7$ years; $59 \%$ were women. The majority of them were recruited from primary care hospitals (81\%). The mean duration of the diagnosis of HT was $8.4 \pm 3.1$ months. There were $1,040(87.8 \%)$ patients on antihypertensive medications. Most of them took one or two agents per day $(39.0 \%$ and $36.1 \%$, resp.). The most commonly used medications were dihydropyridine calcium channel blockers (62.3\%), followed by angiotensin converting enzyme (ACE) inhibitors (45.6\%), while the diuretics were used in $18.6 \%$. The mean $( \pm \mathrm{SD}) \mathrm{BMI}$ in the cohort was $26.5 \pm 5.1 \mathrm{~kg} / \mathrm{m}^{2}$. The mean $( \pm \mathrm{SD})$ clinic and home BP were $143.1 \pm 18.0 / 84.8 \pm 11.7 \mathrm{mmHg}$ and $134.3 \pm 13.9 / 80.6 \pm$ $8.8 \mathrm{mmHg}$, respectively, with the overall white-coat effect (systolic BP/diastolic BP difference between CBP and HBP) of $8.9 \pm 16.4 / 4.2 \pm 9.8 \mathrm{mmHg}$. 
TABLe 1: Demographics and clinical characteristics of the patient population $(n=1,184)$. Values are number $(\%)$ or mean \pm SD.

\begin{tabular}{lc}
\hline Characteristic & Value \\
\hline Age (years) & $58.2 \pm 12.7$ \\
Female & $695(58.7 \%)$ \\
Diabetes & $158(13.3 \%)$ \\
Dyslipidemia & $610(51.5 \%)$ \\
Follow-up at primary care hospital & $960(81.0 \%)$ \\
On antihypertensive therapy & $1,040(87.8 \%)$ \\
Number of antihypertensive medications & \\
1 & $406(39.0 \%)$ \\
2 & $375(36.1 \%)$ \\
$\geq 3$ & $259(24.9 \%)$ \\
Type of antihypertensive medications & \\
Dihydropyridine calcium channel blockers & $648(62.3 \%)$ \\
Angiotensin converting enzyme inhibitors & $474(45.6 \%)$ \\
Diuretics & $193(18.6 \%)$ \\
Others & $636(61.2 \%)$ \\
BMI (kg/m $\left.{ }^{2}\right)$ & $26.5 \pm 5.1$ \\
Clinic BP & \\
Systolic BP (mmHg) & $143.1 \pm 18.0$ \\
Diastolic BP (mmHg) & $84.8 \pm 11.7$ \\
Pulse (beats/min) & $79.2 \pm 12.2$ \\
Home BP & \\
Systolic BP (mmHg) & $134.3 \pm 13.9$ \\
Diastolic BP (mmHg) & $80.6 \pm 8.8$ \\
Pulse (beats/min) & $74.6 \pm 9.9$ \\
Laboratory results & \\
Total cholesterol (mg/dL) & $199.2 \pm 41.2$ \\
Triglyceride (mg/dL) & $152.3 \pm 85.5$ \\
HDL (mg/dL) & $53.9 \pm 14.7$ \\
Calculated LDL (mg/dL) & $268(22.6 \%)$ \\
Creatinine (mg/dL) & \\
GFR <60 mL/min/1.73 m ${ }^{2}$ & \\
\hline
\end{tabular}

BMI; body mass index, BP; blood pressure, HDL; high-density lipoprotein, LDL; low-density lipoprotein, GFR; glomerular filtration rate.

There was no significant difference between nonmedicated and treated patients in all BP components except mean clinic diastolic BP and mean home diastolic BP which were higher in the nonmedicated group $(89.7 \pm 11.1$ vs. $84.1 \pm 11.7 \mathrm{mmHg}$, $p<0.001$, and $82.6 \pm 8.7$ vs. $80.4 \pm 8.8 \mathrm{mmHg}, p=0.004$, resp.).

3.2. Hypertension Subtypes. The prevalence of all $8 \mathrm{HT}$ subtypes is presented in Table 2. Of 144 nonmedicated patients, the prevalence of white-coat HT was $25.7 \%$ with the white-coat effect of $21.2 \pm 4.6 / 10.2 \pm 4.1 \mathrm{mmHg}$ and the prevalence of masked HT was $7.0 \%$. There were 83 patients with sustained HT (57.6\%). Approximately $10 \%$ of nonmedicated participants were found to have normotension since they had normal repeated CBP and mean HBP data. Of 1,040 treated participants, the proportion of patients who had HT with white-coat effect was $23.3 \%$ (white-coat effect of $24.5 \pm 5.7 / 10.1 \pm 6.6 \mathrm{mmHg}$ ). Well-controlled HT, masked uncontrolled HT, and sustained HT were prevalent in $30.0 \%$, $9.6 \%$, and $37.1 \%$ of the treated group, respectively. A total of 486 treated patients $(46.7 \%)$ had uncontrolled HBP (masked uncontrolled HT and sustained HT). There were no differences in baseline characteristics (age, gender, comorbid diseases, and laboratory results) between patients in each HT subtype as summarized in Tables 3 and 4.

3.3. HT Subtypes by Age and Gender. HT subtypes stratified according to age and gender ( $\leq 60$ years and $>60$ years) is shown in Table 5 . In the treated older ( $>60$ years old) subgroups $(n=499)$, uncontrolled HBP was more prevalent in male than female $(53.7 \%$ vs. $42.4 \%, p=0.013)$ but there was no significant difference between genders in younger subgroups $(42.5 \%$ and $48.3 \%, p=0.188)$. The older males had higher prevalence of uncontrolled HBP than younger males $(p=0.02)$ without significant difference in female groups $(p=0.151)$.

3.4. HT Subtype Analyzed by Hospital Regions. Table 6 shows the HT subtype categorized by hospital regions. Most of the patients were enrolled from the Northeast region $(n=276,23.3 \%)$ followed by the Central region $(n=252$, $21.3 \%$ ). The prevalence of HT subtypes was not significantly different according to the hospital regions. Of all five regions, the East region tended to have the highest rate of uncontrolled HBP (53.3\%) followed by the South $(48.8 \%)$ without significant difference when compared with the rest of the country $(p=0.317)$.

\section{Discussion}

This is the first nationwide multicenter study to examine the prevalence of HT subtypes in Thai hypertensives using telemonitoring. We assessed patient characteristics and type of antihypertensive medications and further analyzed subgroup of patients according to gender, age, and geographical regions. In nonmedicated group of the present study, the proportion of patients with white-coat HT and masked HT was $25.7 \%$ and $7.0 \%$, respectively, which was concordant with several published studies. Piper et al. [1] recently conducted a systematic review including studies using HBPM and found a wide range of prevalence of white-coat HT from $16 \%$ to $55 \%$. Stergiou et al. [31] reported the International Database of Home blood pressure in relation to Cardiovascular Outcomes (IDHOCO) study involving 6,458 participants from 5 different populations. They found that $9.8 \%$ of the participants had masked HT. In comparison with the studies using ABPM, Omboni et al. [5] included 14,143 patients from 27 countries and reported the prevalence of white-coat HT and masked HT of $22.6 \%$ and $11.1 \%$, respectively. In the IDACO ABPM registry, white-coat $\mathrm{HT}$ was found in $35.7 \%$ while the prevalence of masked HT was $16.9 \%$ [6]. One possibility of a lower proportion of masked HT in our study is that we defined masked HT using the mean morning and evening HBP values. In contrast with ABPM method, we could not identify the elevated midday $\mathrm{BP}$ and high nocturnal BP during sleep, which are common phenotypes of masked HT [10, 32].

In treated hypertensives, the proportion of patients with HT with white-coat effect and masked uncontrolled HT in the present cohort was $23.3 \%$ and $9.6 \%$, respectively. These 
TABLE 2: Prevalence of eight hypertension (HT) subtypes categorized according to the treatment status, clinic blood pressure, and mean home blood pressure.

\begin{tabular}{|c|c|c|c|}
\hline \multirow{2}{*}{\multicolumn{2}{|c|}{ Nonmedicated patients $(n=144)$}} & \multicolumn{2}{|c|}{ Home blood pressure } \\
\hline & & SBP $<135$ and $\mathrm{DBP}<85 \mathrm{mmHg}$ & $\mathrm{SBP} \geq 135$ and/or $\mathrm{DBP} \geq 85 \mathrm{mmHg}$ \\
\hline \multirow{2}{*}{ Clinic blood pressure } & $\mathrm{SBP}<140$ and $\mathrm{DBP}<90 \mathrm{mmHg}$ & Normotension $14(9.7 \%)$ & Masked HT $10(7.0 \%)$ \\
\hline & $\mathrm{SBP} \geq 1$ & & \\
\hline \multirow{2}{*}{\multicolumn{2}{|c|}{ Treated patients $(n=1,040)$}} & \multicolumn{2}{|c|}{ Home blood pressure } \\
\hline & & SBP $<135$ and $\mathrm{DBP}<85 \mathrm{mmHg}$ & $\mathrm{SBP} \geq 135$ and/or DBP $\geq 85 \mathrm{mmHg}$ \\
\hline Clinic blood pressure & $\begin{array}{l}\mathrm{SBP}<140 \text { and DBP }<90 \mathrm{mmHg} \\
\mathrm{SBP} \geq 140 \text { and/or DBP } \geq 90 \mathrm{mmHg}\end{array}$ & $\begin{array}{c}\text { Well-controlled HT } 312(30.0 \%) \\
\text { HT with white-coat effect } 242(23.3 \%)\end{array}$ & $\begin{array}{c}\text { Masked uncontrolled HT } 100(9.6 \%) \\
\text { Sustained HT } 386(37.1 \%)\end{array}$ \\
\hline
\end{tabular}

SBP; systolic blood pressure, DBP; diastolic blood pressure.

TABLE 3: Patient characteristics categorized by hypertension subtypes in the nonmedicated group.

\begin{tabular}{|c|c|c|c|c|c|c|}
\hline Characteristic & All $(n=144)$ & $\begin{array}{l}\text { Normotension } \\
\quad(n=14)\end{array}$ & $\begin{array}{l}\text { White-coat HT } \\
\qquad(n=37)\end{array}$ & $\begin{array}{l}\text { Masked HT } \\
\quad(n=10)\end{array}$ & $\begin{array}{l}\text { Sustained HT } \\
\quad(n=83)\end{array}$ & $p$ value \\
\hline Age (years) & $53.8 \pm 12.1$ & $51.3 \pm 11.4$ & $53.3 \pm 10.5$ & $54.2 \pm 12.6$ & $53.5 \pm 13.1$ & 0.262 \\
\hline Female & $77(53.5 \%)$ & $7(50 \%)$ & $22(59.5 \%)$ & $6(60.0 \%)$ & $42(50.6 \%)$ & 0.623 \\
\hline Diabetes & $1(0.7 \%)$ & - & $1(2.7 \%)$ & - & - & - \\
\hline Dyslipidemia & $52(36.1 \%)$ & $4(28.6 \%)$ & $12(32.4 \%)$ & $5(50 \%)$ & $25(30.1 \%)$ & 0.351 \\
\hline BMI $\left(\mathrm{kg} / \mathrm{m}^{2}\right)$ & $25.4 \pm 4.4$ & $25.1 \pm 4.2$ & $25.3 \pm 5.4$ & $25.3 \pm 3.2$ & $25.7 \pm 4.0$ & 0.911 \\
\hline \multicolumn{7}{|l|}{ Clinic BP } \\
\hline Systolic BP (mmHg) & $143.2 \pm 16.8$ & $122.4 \pm 7.6$ & $148.4 \pm 8.0$ & $129.4 \pm 7.9$ & $154.3 \pm 12.4$ & $<0.001$ \\
\hline Diastolic BP (mmHg) & $89.7 \pm 11.1$ & $74.8 \pm 7.2$ & $90.1 \pm 6.8$ & $79.5 \pm 6.3$ & $97.1 \pm 9.3$ & $<0.001$ \\
\hline Pulse (beats/min) & $79.2 \pm 11.8$ & $76.1 \pm 8.5$ & $78.8 \pm 10.0$ & $78.7 \pm 5.4$ & $80.9 \pm 11.2$ & 0.531 \\
\hline \multicolumn{7}{|l|}{ Home BP } \\
\hline Systolic BP (mmHg) & $133.0 \pm 12.0$ & $120.2 \pm 8.1$ & $127.3 \pm 5.5$ & $146.6 \pm 6.4$ & $142.0 \pm 8.7$ & 0.435 \\
\hline Diastolic BP (mmHg) & $82.6 \pm 8.7$ & $72.4 \pm 6.6$ & $77.9 \pm 4.6$ & $84.4 \pm 5.3$ & $89.3 \pm 6.3$ & $<0.001$ \\
\hline Pulse (beats/min) & $75.9 \pm 8.7$ & $74.1 \pm 8.7$ & $76.0 \pm 7.7$ & $77.7 \pm 7.4$ & $77.0 \pm 9.1$ & 0.334 \\
\hline \multicolumn{7}{|l|}{ Laboratory results } \\
\hline Total cholesterol (mg/dL) & $210.4 \pm 50.0$ & $221.6 \pm 68.1$ & $211.4 \pm 27.8$ & $212.7 \pm 58.0$ & $202.0 \pm 42.1$ & 0.713 \\
\hline Triglyceride $(\mathrm{mg} / \mathrm{dL})$ & $134.0 \pm 74.9$ & $132.0 \pm 73.0$ & $103.3 \pm 40.5$ & $145.6 \pm 65.0$ & $134.9 \pm 88.3$ & 0.382 \\
\hline $\mathrm{HDL}(\mathrm{mg} / \mathrm{dL})$ & $54.8 \pm 13.7$ & $53.4 \pm 11.3$ & 59. $5 \pm 10.0$ & $51.1 \pm 8.7$ & $56.7 \pm 16.4$ & 0.351 \\
\hline Calculated LDL (mg/dL) & $129.1 \pm 44.4$ & $132.4 \pm 48.2$ & $125.2 \pm 27.3$ & $132.5 \pm 49.1$ & $121.2 \pm 34.0$ & 0.366 \\
\hline Creatinine $(\mathrm{mg} / \mathrm{dL})$ & $0.97 \pm 1.04$ & $0.76 \pm 0.41$ & $0.78 \pm 0.13$ & $0.80 \pm 0.34$ & $0.87 \pm 0.23$ & 0.492 \\
\hline $\begin{array}{l}\mathrm{GFR}<60 \mathrm{~mL} / \mathrm{min} / \\
1.73 \mathrm{~m}^{2}\end{array}$ & $12(15.8 \%)$ & $1(7.1 \%)$ & $2(11.1 \%)$ & $1(16.7 \%)$ & $11(13.3 \%)$ & 0.307 \\
\hline
\end{tabular}

Values are number (\%) or mean \pm SD. The $P$ values reflect comparison between 4 subtypes. $p$ values $<0.05$ are in bold.

numbers are quite similar to $23 \%$ and $9 \%$ reported from the recent AsiaBP@Home study that included the patients from 11 countries across Asia [33]. Comparing with the western study, Stergiou $[34,35]$ et al. reported the prevalence of HT with white-coat effect and masked uncontrolled HT of $22 \%$ and $11.9 \%$, respectively, by using average 2 -visit CBP value and 4-day HBPM value. We further investigated the whitecoat effect across the cohort and found the effect of $8.7 \pm 16.9 / 3.8 \pm 10.0 \mathrm{mmHg}$ in patients receiving antihypertensive medications, while the effect was $10.2 \pm 12.9$ / $7.2 \pm 7.8 \mathrm{mmHg}$ in the nonmedicated patients. These ranges of white-coat effect found in our study are comparable to the previous reports using HBPM $[36,37]$.

In the present study, the controlled HBP was achieved in $53.3 \%$ of treated patients, which is comparable to $51 \%$ from the cross-sectional survey over 25 provinces across Thailand in 2011 [38]. However, this rate is lower than the result from the 2014 Thai National Health Examination Survey $\mathrm{V}$ showing that $60 \%$ of hypertensive participants had controlled BP using field BP target of $<140 / 90 \mathrm{mmHg}$ taken by the community health volunteer home visit [39]. The differences in the patient demographic and BP measurement method could account for the higher BP control rate.

Subgroup analysis of patients according to sex and age found that older males had the least HBP controlled (only $46.4 \%)$, whereas the older females had the most (57.6\%). A recent randomized controlled trial conducted in Thai primary care setting showed that HBPM in older patients significantly decreased the rate of uncontrolled HT from $90 \%$ to $38.2 \%$ in one year, compared with usual care (from $81.8 \%$ to $54.5 \%$ ) [40]. These findings could emphasize the role of HBPM for long-term BP control in the older population, especially in the males.

Regarding the BP control of each geographical region across Thailand, the East region tended to have the greatest rate of uncontrolled HBP whereas the Northeast tended to have the lowest $(57.2 \%$ and $46.7 \%)$. This interregional difference may be attributed to the fact that the Northeast region has the lowest prevalence of HT compared with other 
TABLE 4: Patient characteristics categorized by hypertension subtypes in the treated group.

\begin{tabular}{|c|c|c|c|c|c|c|}
\hline Characteristic & All $(n=1,040)$ & $\begin{array}{l}\text { Well-controlled } \\
\text { HT }(n=312)\end{array}$ & $\begin{array}{l}\text { HT with white-coat } \\
\text { effect }(n=242)\end{array}$ & $\begin{array}{l}\text { Masked uncontrolled } \\
\text { HT }(n=100)\end{array}$ & $\begin{array}{c}\text { Sustained } \\
\text { HT }(n=386)\end{array}$ & $p$ value \\
\hline Age (years) & $59.0 \pm 12.5$ & $58.9 \pm 12.5$ & $58.5 \pm 12.3$ & $59.3 \pm 12.5$ & $59.2 \pm 12.6$ & 0.907 \\
\hline Female & $618(59 \%)$ & $195(62.5 \%)$ & $140(57.9 \%)$ & $54(54.0 \%)$ & $229(59.3 \%)$ & 0.441 \\
\hline Diabetes & $157(15.1 \%)$ & $37(11.8 \%)$ & $38(15.7 \%)$ & $14(14.0 \%)$ & $68(17.6 \%)$ & 0.362 \\
\hline Dyslipidemia & $558(53.7 \%)$ & $169(54.2 \%)$ & $131(54.1 \%)$ & $52(52 \%)$ & $206(53.4 \%)$ & 0.854 \\
\hline BMI $(\mathrm{kg} / \mathrm{m} 2)$ & $26.6 \pm 5.2$ & $26.3 \pm 4.5$ & $26.7 \pm 4.9$ & $26.7 \pm 4.7$ & $26.8 \pm 5.9$ & 0.717 \\
\hline \multicolumn{7}{|l|}{ Clinic BP } \\
\hline Systolic BP (mmHg) & $143.1 \pm 18.1$ & $125.7 \pm 9.0$ & $150.8 \pm 11.3$ & $127.8 \pm 9.1$ & $156.3 \pm 14.2$ & $<0.001$ \\
\hline Diastolic BP (mmHg) & $84.1 \pm 11.7$ & $76.45 \pm 7.6$ & $87.4 \pm 9.8$ & $76.7 \pm 8.4$ & $90.2 \pm 11.7$ & $<0.001$ \\
\hline Pulse (beats/min) & $79.2 \pm 12.2$ & $77.61 \pm 10.3$ & $80.6 \pm 13.3$ & $77.8 \pm 12.0$ & $79.9 \pm 12.9$ & 0.012 \\
\hline \multicolumn{7}{|l|}{ Home BP } \\
\hline Systolic BP (mmHg) & $134.4 \pm 14.2$ & $122.9 \pm 6.9$ & $125.8 \pm 6.3$ & $145.5 \pm 8.1$ & $147.1 \pm 11.3$ & $<0.001$ \\
\hline Diastolic BP (mmHg) & $80.4 \pm 8.8$ & $74.6 \pm 5.6$ & $76.0 \pm 5.6$ & $84.9 \pm 6.4$ & $86.6 \pm 8.3$ & $<0.001$ \\
\hline Pulse (beats/min) & $74.4 \pm 10.1$ & $73.9 \pm 8.9$ & $74.1 \pm 10.0$ & $75.1 \pm 10.9$ & $74.7 \pm 10.9$ & 0.599 \\
\hline \multicolumn{7}{|l|}{ Laboratory results } \\
\hline Total cholesterol (mg/dL) & $198.2 \pm 40.2$ & $196.3 \pm 36.9$ & $199.1 \pm 42.2$ & $196.0 \pm 42.1$ & $200.0 \pm 41.2$ & 0.678 \\
\hline Triglyceride $(\mathrm{mg} / \mathrm{dL})$ & $153.8 \pm 86.2$ & $148.0 \pm 86.4$ & $157.3 \pm 86.4$ & $153.5 \pm 72.8$ & $156.6 \pm 89.1$ & 0.603 \\
\hline $\mathrm{HDL}(\mathrm{mg} / \mathrm{dL})$ & $53.3 \pm 14.8$ & $54.0 \pm 13.2$ & $53.6 \pm 15.3$ & $52.1 \pm 12.5$ & $52.9 \pm 16.4$ & 0.731 \\
\hline Calculated LDL (mg/dL) & $113.9 \pm 37.8$ & $112.3 \pm 33.9$ & $113.8 \pm 41.1$ & $112.0 \pm 39.6$ & $115.9 \pm 38.5$ & 0.687 \\
\hline Creatinine $(\mathrm{mg} / \mathrm{dL})$ & $0.94 \pm 0.50$ & $0.89 \pm 0.28$ & $0.90 \pm 0.32$ & $0.92 \pm 0.35$ & $1.02 \pm 0.71$ & 0.007 \\
\hline $\mathrm{GFR}<60 \mathrm{~mL} / \mathrm{min} / 1.73 \mathrm{~m}^{2}$ & $256(28.2 \%)$ & $76(27.3 \%)$ & $54(26.6 \%)$ & $23(26.4 \%)$ & $101(30.1 \%)$ & 0.802 \\
\hline
\end{tabular}

Values are number (\%) or mean \pm SD. The $p$ values reflect comparison between 4 subtypes. $p$ values $<0.05$ are in bold. Abbreviations as Table 1 .

TABLE 5: Prevalence of hypertension (HT) subtypes and BP control patterns further classified according to gender and age ( $\leq 60$ years and $>60$ years).

\begin{tabular}{|c|c|c|c|c|c|c|}
\hline & \multicolumn{2}{|c|}{ Male } & \multirow{2}{*}{$p$ value } & \multicolumn{2}{|c|}{ Female } & \multirow{2}{*}{$p$ value } \\
\hline & Age $\leq 60$ years & Age $>60$ years & & Age $\leq 60$ years & Age $>60$ years & \\
\hline Nonmedicated patient $(n=144)$ & $53(100 \%)$ & $16(100 \%)$ & \multirow{5}{*}{0.604} & $55(100 \%)$ & $20(100 \%)$ & \multirow{5}{*}{0.565} \\
\hline Normotension & $4(7.5 \%)$ & $3(18.7 \%)$ & & $5(9.1 \%)$ & $2(10.0 \%)$ & \\
\hline White-coat HT & $10(18.9 \%)$ & $5(31.2 \%)$ & & $18(32.7 \%)$ & $4(20.0 \%)$ & \\
\hline Masked HT & $3(5.7 \%)$ & $1(6.3 \%)$ & & $5(9.1 \%)$ & $1(5.0 \%)$ & \\
\hline Sustained HT & $36(67.9 \%)$ & $7(43.8 \%)$ & & $27(49.1 \%)$ & $13(65.0 \%)$ & \\
\hline Treated patient $(n=1,040)$ & $212(100 \%)$ & $216(100 \%)$ & \multirow{5}{*}{0.138} & $329(100 \%)$ & $283(100 \%)$ & \multirow{5}{*}{0.230} \\
\hline Well-controlled HT & $64(30.1 \%)$ & $55(25.5 \%)$ & & $95(28.8 \%)$ & $101(35.7 \%)$ & \\
\hline HT with white-coat effect & $58(27.4 \%)$ & $45(20.8 \%)$ & & $75(22.8 \%)$ & $62(21.9 \%)$ & \\
\hline Masked uncontrolled HT & $19(9.0 \%)$ & $27(12.5 \%)$ & & $27(8.2 \%)$ & $27(9.5 \%)$ & \\
\hline Sustained HT & $71(33.5 \%)$ & $89(41.2 \%)$ & & $132(40.2 \%)$ & $93(32.9 \%)$ & \\
\hline Controlled $\mathrm{HBP}^{\mathrm{a}}$ & $122(57.5 \%)$ & $100(46.3 \%)$ & \multirow{2}{*}{0.020} & $170(51.7 \%)$ & $163(57.6 \%)$ & \multirow{2}{*}{0.151} \\
\hline Uncontrolled $\mathrm{HBP}^{\mathrm{b}}$ & $90(42.5 \%)$ & $116(53.7 \%)^{*}$ & & $159(48.3 \%)$ & $120(42.4 \%)^{*}$ & \\
\hline
\end{tabular}

Values are number. ${ }^{a}$ Controlled HBP included well-controlled HT and HT with white-coat effect. ${ }^{b}$ Uncontrolled HBP included masked uncontrolled HT and sustained HT in treated patients. ${ }^{*} p$ value between male age $>60$ years and female age $>60$ years $=0.013$.

regions [39], the difference in healthcare systems, and high salt intake in the East region. More importantly, this data should prompt local healthcare authorities for further evaluation and action to improve HT care.

Telehealth technology has been strongly recommended in the recent guidelines for the prevention, detection, and management of high $\mathrm{BP}$ in adults $[11,15]$. It can be implemented with adjunct active interventions from healthcare providers such as the titration of medication or giving feedback to the patients or it can be used as only passive telemonitoring $[41,42]$ as demonstrated in our study. A recent meta-analysis of randomized controlled studies showed that the effect of home telemonitoring on BP control was greater than that of BP self-monitoring without transmission of HBP data [21]. This emphasizes an incremental value of the teletransmission approach to minimize self-reporting bias [23]. The present study shows that implementing telehealth-assisted HBPM technology in Thailand was feasible. We constructed a network of hypertensive care across all regions and provided Internetbased online database. Achieving target BP control required monitoring and an excellent standard of care. Thus, our study could be the first step to enhance the role of technological advances for BP control in Thailand. The ongoing 1-year follow-up study of THAI HBPM to examine the BP control rate after implementing the HBPM-facilitated medication titration could further highlight the role of telemonitoring in the management of HT. 
TABLE 6: Prevalence of hypertension (HT) subtypes and BP control patterns further classified according to the hospital regions of Thailand.

\begin{tabular}{|c|c|c|c|c|c|c|}
\hline & North & Northeast & East & Central & South & $p$ value \\
\hline Nonmedicated patient $(n=144)$ & $7(100 \%)$ & $12(100 \%)$ & $80(100 \%)$ & $34(100 \%)$ & $11(100 \%)$ & \multirow{5}{*}{0.056} \\
\hline Normotension & $2(28.5 \%)$ & $2(16.7 \%)$ & $6(7.5 \%)$ & $3(8.8 \%)$ & $1(9.1 \%)$ & \\
\hline White-coat HT & $1(14.3 \%)$ & $4(33.3 \%)$ & $23(28.8 \%)$ & $7(20.6 \%)$ & $2(18.2 \%)$ & \\
\hline Masked HT & 0 & $1(8.4 \%)$ & $5(6.2 \%)$ & $1(2.9 \%)$ & $3(27.3 \%)$ & \\
\hline Sustained HT & $4(57.1 \%)$ & $5(41.6 \%)$ & $46(57.5 \%)$ & $23(67.7 \%)$ & $5(45.4 \%)$ & \\
\hline Treated patient $(n=1,040)$ & $240(100 \%)$ & $264(100 \%)$ & $150(100 \%)$ & $218(100 \%)$ & $168(100 \%)$ & \multirow{5}{*}{0.197} \\
\hline Well-controlled HT & $82(34.2 \%)$ & $90(34.1 \%)$ & $32(21.3 \%)$ & $57(26.1 \%)$ & $51(30.4 \%)$ & \\
\hline HT with white-coat effect & $49(20.4 \%)$ & $61(23.1 \%)$ & $38(25.3 \%)$ & $59(27.1 \%)$ & $35(20.8 \%)$ & \\
\hline Masked uncontrolled HT & $25(10.4 \%)$ & $22(8.3 \%)$ & $15(10.1 \%)$ & $25(11.5 \%)$ & $13(7.7 \%)$ & \\
\hline Sustained HT & $84(35.0 \%)$ & $91(34.5 \%)$ & $65(43.3 \%)$ & $77(35.3 \%)$ & $69(41.1 \%)$ & \\
\hline Controlled $\mathrm{HBP}^{\mathrm{a}}$ & $131(54.6 \%)$ & $151(57.2 \%)$ & $70(46.7 \%)$ & $116(53.2 \%)$ & $86(51.2 \%)$ & \multirow{2}{*}{0.317} \\
\hline Uncontrolled $\mathrm{HBP}^{\mathrm{b}}$ & $109(45.4 \%)$ & $113(42.8 \%)$ & $80(53.3 \%)$ & $102(46.8 \%)$ & $82(48.8 \%)$ & \\
\hline
\end{tabular}

Values are number. The $p$ values reflect the comparison between 5 regions. ${ }^{a}$ Controlled HBP included well-controlled HT and HT with white-coat effect.

${ }^{b}$ Uncontrolled HBP included masked uncontrolled HT and sustained HT in treated patients.

The present study has some limitations. Firstly, the majority of the participants were enrolled from primary care hospitals and managed by general practitioners; thus the results may not be generalizable to the larger scale hospitals. However, our finding can still represent the characteristic of HT subtypes in real-life practice since the vast majority of Thai hypertensives have been followed at primary care centers [40]. Secondly, the proportion of patients with white-coat and masked HT may not be accurate since the number of patients in the nonmedicated group was small. Moreover, this study enrolled the participants solely based on high CBP; thus the majority of patients with masked HT who have normal or borderline CBP may have not been included. Thirdly, since the data on current smoking status is not available, this could affect the characteristic of masked HT. The out-of-clinic smoking potentially affects the raising of BP at home [32]. Lastly, due to the fact that this study was conducted in various clinics across the country, thus CBP data were obtained from different sphygmomanometers' models. However, we minimized intraobserver and interobserver variations by training the staff to perform CBP and clinical validation in the same manner according to standard guideline recommendations $[2,26,27]$. It is noteworthy that the strength of our study is that we used a single model of validated HBP device for all study sites, which resulted in highly robust HBP data. Moreover, we implemented the cloud-based transmission of HBP data that overcomes the self-reporting bias, which is a limitation of conventional HBPM in real-world practice.

\section{Conclusions}

This is the first nationwide study in Thailand to demonstrate the prevalence and characteristics of HT subtypes in Thai hypertensives using telemonitoring. Almost one-fourth had white-coat HT or HT with white-coat effect. Approximately half of the treated hypertensives, especially in the older males, had uncontrolled HBP which requires more intensive interventions. The cost-effectiveness of utilizing THAI HBPM for long-term BP control in routine practice needs to be examined in the future study.

\section{Data Availability}

The related data used to support the results of this study are available from the corresponding author upon request.

\section{Conflicts of Interest}

All authors declare no conflicts of interest.

\section{Acknowledgments}

The authors gratefully acknowledge investigators, staff, and participants of all 46 sites for their contribution to this study. They are thankful to Naiyana Kanjanapibul RN and Sawangchit Kongpibarn RN who are the nurse coordinators. In addition, they would like to thank the Ministry of Public Health of Thailand, the Heart Association of Thailand, and the Thai Hypertension Society for their promotion and support of this Nationwide project. The major source of funding was from the charity concert as a fundraising event held by the Division of Cardiovascular Medicine, Department of Medicine, Faculty of Medicine, Chulalongkorn University to commemorate Her Majesty the Queen's $84^{\text {th }}$ Birthday Anniversary Celebrations on the $12^{\text {th }}$ of August 2016. This study was also supported in part by the Heart Association of Thailand and the Thai Hypertension Society.

\section{Supplementary Materials}

Supplementary list of all 46 participating sites. Supplementary Figure 1: specification of the oscillometric home blood pressure monitoring device used in the study (Uright model TD-3128, TaiDoc Technology Corporation, Taiwan). Supplementary Figure 2: patient enrollment flow chart of the study. Supplementary Table 1: clinical validation of home blood pressure device according to British Hypertension Society grading criteria. (Supplementary Materials)

\section{References}

[1] M. A. Piper, C. V. Evans, B. U. Burda, K. L. Margolis, E. O'Connor, and E. P. Whitlock, "Diagnostic and predictive 
accuracy of blood pressure screening methods with consideration of rescreening intervals: a systematic review for the U.S. preventive services task force," Annals of Internal Medicine, vol. 162, no. 3, pp. 192-204, 2015.

[2] G. Mancia, R. Fagard, K. Narkiewicz et al., "2013 ESH/ESC guidelines for the management of arterial hypertension: the task force for the management of arterial hypertension of the European society of hypertension (ESH) and of the European society of cardiology (ESC)," European Heart Journal, vol. 34, no. 28, pp. 2159-2219, 2013.

[3] P. K. Whelton, R. M. Carey, W. S. Aronow et al., "2017 ACC/ AHA/AAPA/ABC/ACPM/AGS/APhA/ASH/ASPC/NMA/ PCNA guideline for the prevention, detection, evaluation, and management of High blood pressure in adults: executive summary: a report of the American college of cardiology/ American heart association task force on clinical Practice guidelines," Hypertension, vol. 72, no. 3, p. e33, 2018.

[4] R. Agarwal, J. E. Bills, T. J. W. Hecht, and R. P. Light, "Role of home blood pressure monitoring in overcoming therapeutic inertia and improving hypertension control," Hypertension, vol. 57, no. 1, pp. 29-38, 2011.

[5] S. Omboni, D. Aristizabal, A. De la Sierra et al., "Hypertension types defined by clinic and ambulatory blood pressure in 14 143 patients referred to hypertension clinics worldwide. Data from the ARTEMIS study," Journal of Hypertension, vol. 34, no. 11, pp. 2187-2198, 2016.

[6] J. D. Melgarejo, G. E. Maestre, L. Thijs et al., "Prevalence, treatment, and control rates of conventional and ambulatory hypertension across 10 populations in 3 continents," $\mathrm{Hy}$ pertension, vol. 70, no. 1, pp. 50-58, 2017.

[7] T. G. Pickering, G. D. James, C. Boddie, G. A. Harshfield, S. Blank, and J. H. Laragh, "How common is white coat hypertension?" JAMA: The Journal of the American Medical Association, vol. 259, no. 2, pp. 225-228, 1988.

[8] T. Ohkubo, M. Kikuya, H. Metoki et al., "Prognosis of "masked" hypertension and "White-Coat" hypertension detected by 24 -h ambulatory blood pressure monitoring," Journal of the American College of Cardiology, vol. 46, no. 3, pp. 508-515, 2005.

[9] D. Conen, S. Aeschbacher, L. Thijs et al., "Age-specific differences between conventional and ambulatory daytime blood pressure values," Hypertension, vol. 64, no. 5, pp. 1073-1079, 2014.

[10] T. Fujiwara, Y. Yano, S. Hoshide, H. Kanegae, and K. Kario, "Association of cardiovascular outcomes with masked hypertension defined by home blood pressure monitoring in a Japanese general practice population," JAMA Cardiology, vol. 3, no. 7, pp. 583-590, 2018.

[11] P. K. Whelton, R. M. Carey, W. S. Aronow et al., "2017 ACC/ AHA/AAPA/ABC/ACPM/AGS/APhA/ASH/ASPC/NMA/ $\mathrm{PCNa}$ guideline for the prevention, detection, evaluation, and management of high blood pressure in adults: a report of the American college of cardiology/American heart association task force on clinical practice guidelines," Journal of the American College of Cardiology, vol. 71, no. 19, pp. e127-e248, 2018.

[12] M. Satoh, T. Maeda, S. Hoshide, and T. Ohkubo, "Is antihypertensive treatment based on home blood pressure recommended rather than that based on office blood pressure in adults with essential hypertension? (meta-analysis)," Hypertension Research, vol. 42, no. 6, pp. 807-816, 2019.

[13] B. Williams, G. Mancia, W. Spiering et al., "2018 ESC/ESH guidelines for the management of arterial hypertension," Journal of Hypertension, vol. 36, no. 10, pp. 1953-2041, 2018.
[14] P. Muntner, D. Shimbo, R. M. Carey et al., "Measurement of blood pressure in humans: a scientific statement from the American heart association," Hypertension, vol. 73, no. 5, pp. e35-e66, 2019.

[15] S. Umemura, H. Arima, S. Arima et al., "The Japanese society of hypertension guidelines for the management of hypertension (JSH 2019)," Hypertension Research, vol. 42, no. 9, pp. 1235-1481, 2019.

[16] D. Shimbo, M. Abdalla, L. Falzon, R. R. Townsend, and P. Muntner, "Role of ambulatory and home blood pressure monitoring in clinical practice," Annals of Internal Medicine, vol. 163, no. 9, pp. 691-700, 2015.

[17] A. J. Viera, A. L. Hinderliter, A. V. Kshirsagar, J. Fine, and R. Dominik, "Reproducibility of masked hypertension in adults with untreated borderline office blood pressure: comparison of ambulatory and home monitoring," American Journal of Hypertension, vol. 23, no. 11, pp. 1190-1197, 2010.

[18] Y. Imai, T. Obara, K. Asamaya, and T. Ohkubo, "The reason why home blood pressure measurements are preferred over clinic or ambulatory blood pressure in Japan," Hypertension Research, vol. 36, no. 8, pp. 661-672, 2013.

[19] M. A. Weber, "The evolving clinical management of hypertension," The Journal of Clinical Hypertension, vol. 16, no. 12, pp. 917-924, 2014.

[20] K. L. Margolis, S. E. Asche, A. R. Bergdall et al., "Effect of home blood pressure telemonitoring and pharmacist management on blood pressure control," JAMA, vol. 310, no. 1, pp. 46-56, 2013.

[21] S. Omboni, T. Gazzola, G. Carabelli, and G. Parati, "Clinical usefulness and cost effectiveness of home blood pressure telemonitoring," Journal of Hypertension, vol. 31, no. 3, pp. 455-468, 2013.

[22] W. J. Verberk, A. G. H. Kessels, and T. Thien, "Telecare is a valuable tool for hypertension management, a systematic review and meta-analysis," Blood Pressure Monitoring, vol. 16, no. 3, pp. 149-155, 2011.

[23] M. G. Myers and G. S. Stergiou, "Reporting bias: achilles' heel of home blood pressure monitoring," Journal of the American Society of Hypertension, vol. 8, no. 5, pp. 350-357, 2014.

[24] E. O’Brien, R. Asmar, L. Beilin et al., "European society of hypertension recommendations for conventional, ambulatory and home blood pressure measurement," Journal of Hypertension, vol. 21, no. 5, pp. 821-848, 2003.

[25] M. A. Weber, E. L. Schiffrin, W. B. White et al., "Clinical practice guidelines for the management of hypertension in the community," The Journal of Clinical Hypertension, vol. 16, no. 1, pp. 14-26, 2014.

[26] E. O’Brien, T. Pickering, R. Asmar et al., "Working group on blood pressure monitoring of the European society of hypertension international protocol for validation of blood pressure measuring devices in adults," Blood Pressure Monitoring, vol. 7, no. 1, pp. 3-17, 2002.

[27] G. S. Stergiou, N. Karpettas, N. Atkins, and E. O’Brien, "European society of hypertension international protocol for the validation of blood pressure monitors: a critical review of its application and rationale for revision," Blood Pressure Monitoring, vol. 15, no. 1, pp. 39-48, 2010.

[28] K. Kario, S. Park, P. Buranakitjaroen et al., "Guidance on home blood pressure monitoring: a statement of the HOPE Asia network," The Journal of Clinical Hypertension, vol. 20, no. 3, pp. 456-461, 2018.

[29] http://www.accessdata.fda.gov/scripts/cdrh/cfdocs/cfpmn/pmn. cfm?ID=K112216. 
[30] K. Kario, N. Tomitani, P. Buranakitjaroen et al., "Rationale and design for the Asia BP@Home study on home blood pressure control status in 12 Asian countries and regions," The Journal of Clinical Hypertension, vol. 20, no. 1, pp. 33-38, 2018.

[31] G. S. Stergiou, K. Asayama, L. Thijs et al., "Prognosis of whitecoat and masked hypertension," Hypertension, vol. 63, no. 4, pp. 675-682, 2014.

[32] S. S. Franklin, E. O’Brien, and J. A. Staessen, "Masked hypertension: understanding its complexity," European Heart Journal, vol. 38, no. 15, pp. 1112-1118, 2017.

[33] K. Kario, N. Tomitani, P. Buranakitjaroen et al., "Home blood pressure control status in 2017-2018 for hypertension specialist centers in Asia: results of the Asia BP@Home study," The Journal of Clinical Hypertension, vol. 20, no. 12, pp. 1686-1695, 2018.

[34] G. Stergiou, S. P. Efstathiou, C. K. Argyraki, L. G. Roussias, and T. D. Mountokalakis, "White coat effect in treated versus untreated hypertensive individuals: a case-control study using ambulatory and home blood pressure monitoring," American Journal of Hypertension, vol. 17, no. 2, pp. 124-128, 2004.

[35] G. Stergiou, E. Salgami, D. Tzamouranis, and L. Roussias, "Masked hypertension assessed by ambulatory blood pressure versus home blood pressure monitoring: is it the same phenomenon?" American Journal of Hypertension, vol. 18, no. 6, pp. 772-778, 2005.

[36] A. R. Feinstein, "On white-coat effects and the electronic monitoring of compliance," Archives of Internal Medicine, vol. 150, no. 7, pp. 1377-1378, 1990.

[37] P. Verdecchia, "White-coat hypertension in adults and children," Blood Pressure Monitoring, vol. 4, no. 3-4, pp. 175-179, 1999.

[38] P. Buranakitjaroen, "Hypertension audit in clinical practice based in Thailand (HABIT)," Journal of the Medical Association of Thailand, vol. 94, no. Suppl 1, pp. S57-S65, 2011.

[39] Y.-C. Chia, P. Buranakitjaroen, C.-H. Chen et al., "Current status of home blood pressure monitoring in Asia: statement from the HOPE Asia network," The Journal of Clinical Hypertension, vol. 19, no. 11, pp. 1192-1201, 2017.

[40] W. Aekplakorn, P. Suriyawongpaisal, R. Tansirisithikul, T. Sakulpipat, and P. Charoensuk, "Effectiveness of selfmonitoring blood pressure in primary care," Journal of Primary Care \& Community Health, vol. 7, no. 2, pp. 58-64, 2016.

[41] C. J. Lee and S. Park, "The role of home blood pressure telemonitoring for blood pressure control," Pulse, vol. 4, no. 23, pp. 78-84, 2016.

[42] R. J. McManus, J. Mant, M. Franssen et al., "Efficacy of selfmonitored blood pressure, with or without telemonitoring, for titration of antihypertensive medication (TASMINH4): an unmasked randomised controlled trial," The Lancet, vol. 391, no. 10124, pp. 949-959, 2018. 